\title{
Clinical management of Sialadenitis
}

SADJ September 2020, Vol. 75 No. 8 p11- p412

N Kana', Z Cassim², S Maharaj ${ }^{3}$

\section{INTRODUCTION}

Within the area of salivary gland pathology, obstructive sialadenitis is the most common inflammatory condition of the salivary glands. ${ }^{1}$

It has been well established in the literature that salivary calculi occur most commonly in the submandibular gland, whereas fewer cases are found in the parotid gland, while the sublingual gland and the minor salivary glands form no more than $2 \%$ of cases. ${ }^{2}$

The early treatment of sialadenitis is usually conservative and involves hydration, anti inflammatory medication in conjunction to antibiotics when a bacterial infection is suspected. However, when initial treatment fails, further intervention is needed.

The traditional external approach is sialadenectomy. However, with this exists the potential for injury to the lingual and facial nerves. Further complications including bleeding, infection and an unsightly scar are also found with this procedure. ${ }^{3,4}$

Sialendoscopy is a relatively new technique that only became available once optics had improved to the extent that fiber-optic endoscopes could be miniaturized to a diameter of $0.9 \mathrm{~mm}$ to $1.6 \mathrm{~mm}$. This has ushered in a new era for the management of sialadenitis, particularly in cases where sialadenitis was caused by salivary duct obstruction.

It must be noted that in South Africa, there are currently no generally accepted guidelines on the management of sialadenitis secondary to salivary ductal obstruction as well as in the role of sialendoscopy within the treatment algorithm.

\section{Author affiliations:}

1. Nadir Kana: $M B C h B, M M E D, F C O R L(S A)$, Otorhinolaryngologist in private practice at Netcare Milpark Hospital, Johannesburg, South Africa.

ORCID Number: 0000-0001-8902-2684

2. Zarreen Cassim: BA (Speech Pathology and Audiology), MA (Audiology), University of the Witwatersrand, Johannesburg, South Africa, Johannesburg South Africa, South Africa.

3. Shivesh Maharaj: MBBCH, MMED, FCORL (SA), Current Head of Academic Department, Department of Otorhinolaryngology, University of the Witwatersrand, Johannesburg, South Africa.

Corresponding author: Nadir Kana

Otorhinolaryngologist in private practice at Netcare Milpark Hospital, 9 Guild Road, Parktown, Johannesburg, South Africa.

Email: nadirkana@gmail.com

Author contributions:

1. Nadir Kana: Conceptualisation, write up, editing - $40 \%$

2. Zarreen Cassim: Write up and editing - 30\%

3. Shivesh Maharaj: Conceptualisation and write up - $30 \%$

\section{METHODS}

Currently sialendoscopy is now the benchmark against which radiological tests are measured. As often happens in clinical medicine, the rather invasive nature of sialadenectomy ushered in the need for an alternative approach.

For the purpose of this communication, the researchers, including two Ear Nose and Throat surgeons, reviewed the trends within the setting of their personal clinical practice, over a period of 12 years, commencing with the year 2008. It must be noted that the researchers' experience with sialendoscopy has not only been positive but appears to be largely supportive of the findings reflected in the international literature. This report involved a review of the clinical experience of two surgeons and reflected an $86 \%$ success rate when sialendoscopy was used as a management tool, and a $100 \%$ success rate when it was utilized as a diagnostic tool. Thus far, all the cases seen by the authors have been adult patients, with all of the procedures performed under general anaesthesia.

\section{DISCUSSION}

The first successful diagnostic sialendoscope was performed by Katz et al. in 1990. ${ }^{5}$ As technology in optics improved it became possible not only to diagnose salivary duct obstructions, but a hollow working channel in the center of the endoscope allowed for the passage of specifically designed tools such as hand drills, stone removal baskets and later, fibre lasers. A further development was the successful use of lithotripsy for the fragmentation of large salivary calculi. However, it must be noted that salivary ductal obstruction does not only occur due to calculi.

Salivary strictures versus calculi occur with approximately a 20/80 ratio split. Short strictures are referred to as stenoses and are more easily dilated via sialendoscopy than their longer counterparts. ${ }^{6}$ Currently, sialendoscopy is the most sensitive diagnostic tool used, in comparison to radiological imaging methods.

The diagnosis of sialolithiasis, stenosis, polyps, recurrent sialadenitis, foreign bodies and sialadenosis are made with ease using the sialendoscope. The advantage of sialendoscopy is its ability to treat ductal obstruction. The passage of the sialendoscope itself through salivary ducts dilates minimally stenosed ducts. In addition to this, high-pressure saline solution aids in the dilatory process. The need for sialendoscopy was largely due to the complications associated with invasive and open procedures such as the sialadenectomy, which resulted in the presence of a visible scar. 
Furthermore with the submandibulectomy procedure there are potential complications including dysgeusia, partial tongue paralysis and marginal mandibular nerve palsy (although this is more often a temporary paresis). Parotidectomy is associated with higher risks such as facial nerve injury which is the foremost risk, followed by Frey's Syndrome. ${ }^{7}$ Sialendoscopy itself is minimally invasive, however, it is not entirely complication free, and can rarely be associated with duct avulsion especially during active sialadenitis. Thus acute sialadenitis is a universally accepted contraindication to Sialendoscopy.

Other complications include failed extraction of a salivary calculus, and excessive bleeding leading to abandonment of the procedure. In some cases, the procedure would be repeated but in others a decision to proceed to open adenectomy may be taken.

A further variation of the procedure is a combined external/sialendoscopic procedure which is indicated particularly in cases where the calculi are unusually large. The basic sialendoscopy procedure falls into three steps: the first of which involves the papillary dilation, followed by the passing of the sialendoscope, diagnosis, and lastly, the treatment of the obstruction. The papilla is more readily located using a microscope or magnifying loupes. Furthermore the papilla can be exposed by massaging the gland to initiate salivary flow.

A sialagogue such may aid this technique, for example, the use of lemon juice. The diameter of the undilated papilla is about $0.5 \mathrm{~mm}$ necessitating the use of ductal dilation in order to accommodate a working channel. This is as a therapeutic scope has a diameter of $1.3 \mathrm{~mm}$ to $1.6 \mathrm{~mm}$. The "classic technique" to dilate the papilla is with the use of salivary probes. These are similar to lacrimal dilators. A conical dilator that is less traumatic to the ductal lumen can also be used instead of salivary probes. This is possible only when the papilla opening is large and clearly visible.

The "guided puncture technique" begins with the introduction of probes of increasing size followed by a guidewire. A conical dilator is 'railroaded' over this guide to expand the papilla. The dilatator is then removed and the endoscope working channel is once again 'railroaded' over the guidewire.

The guide is removed when a ductal image is correctly obtained. In cases where the submandibular duct papilla is difficult to find, a more invasive "surgical" technique can be useful. An incision is made parallel to the course of the duct. The duct is then identified and incised by $1 \mathrm{~mm}$ to allow the insertion of the endoscope. In terms of the location of stones, for mobile stones less than $5 \mathrm{~mm}$ located in the distal duct/ papilla, sialendoscopy with calculus retrieval via stone basket may be attempted. If located in the proximal duct/hilum, in the case of small, mobile calculi less than $5 \mathrm{~mm}$, retrieval of the calculus with a wire basket or grasping forceps is indicated. In the case of calculi that are greater than $7 \mathrm{~mm}$ which are palpable, the stones can be fragmented using laser, lithotripsy or a transoral incision of the duct can be performed. Intraparenchymal, mobile stones less than $7 \mathrm{~mm}$ can be removed using the sialendoscope. With impacted calculi greater than $7 \mathrm{~mm}$ up to $10 \mathrm{~mm}$, fragmentation is recommended, thus allowing for endoscopic removal. With regards to the use of the basket, there are various retrieval baskets that are now available to remove calculi. Since the advent of the stone basket, less damage to the ducts and glandular parenchyma have been reported. When a laser is used, the main limiting factor for sialendoscopy in sialolithiasis is the size of calculi. The different techniques described for calculus fragmentation include external lithotripsy, electrohydraulic, piezoelectric, electromagnetic and pneumoblastic lithotripsy and holmium: YAG lithotripsy.

\section{CONCLUSION}

Based on the information presented above, it can be seen that within a clinical context, sialendoscopy can be used dynamically by practitioners not only as a diagnostic tool, but as a therapeutic measure. In the management of salivary duct obstructions specifically, endoscopes with working channels allow for concomitant use of instrumentation to assist in sialolith removal or stricture dilation. In some centres, lithotripsy may be used to facilitate stone fragmentation prior to removal. For stones not amenable to endoluminal removal, a combined approach using a limited incision in conjunction with sialendoscopy to localize and stabilize the stone can provide minimal surgical morbidity, as is the case for complex strictures/dilatations of the duct. ${ }^{4,5}$

The success rates of sialendoscopy vary between $85 \%$ and $95 \%$ which has resulted in a drastic reduction in the need for invasive procedures such as sialadenectomy. ${ }^{6}$ Thus, sialendoscopy has a major role to play in the diagnosis and management of sialolithiasis and such a field warrants more research, especially due to the success that practitioners have seen with regards to the use of the procedure within clinical practice.

\section{References}

1. Strychowsky JE, Sommer DD, Gupta MK, Cohen N, Nahlieli O. Sialendoscopy for the management of obstructive salivary gland disease: a systematic review and meta-analysis. Arch Otolaryngol Head Neck Surg. 138(6): 541-7.

2. lanovski I, Morton RP, Ahmad Z. Patient-perceived outcome after sialendoscopy using the Glasgow benefit inventory. Laryngoscope. 2014; 124(4): 869-74. doi:10.1002/lary.24343.

3. Kroll T, Finkensieper M, Sharma SJ, Guntinas-Lichius O, Wittekindt C. Short-term outcome and patient satisfaction after sialendoscopy. Eur Arch Otorhinolaryngol. 2013; 270(11): 2939- 45.

4. Marchal F, Dulguerov P. Sialolithiasis management: the state of the art. Arch Otolaryngol Head Neck Surg. 2003; 129: 951-6.

5. Nahlieli O, Nakar LH, Nazarian Y, Turner MD. Sialoendoscopy: a new approach to salivary gland obstructive pathology. J Am Dent Assoc. 2006; 137(10): $1394-400$.

6. Katz P. Endoscopy of the salivary glands. Ann Radiol (Paris) [cited 2018 Oct 6]. 1991; 34(1-2): 110-3. Available from: http://www.ncbi.nlm.nih.gov/pubmed/1897843.

7. Singh PP, Gupta V. Sialendoscopy: introduction, indications and technique. Indian J Otolaryngol Head Neck Surg. 2014; 66: 74-8.

8. Nahlieli O. Complications of sialendoscopy: personal experience, literature analysis, and suggestions. J Oral Maxillofac Surg. 2015; 73. 